\title{
Enhanced Extraction Technique of Omarigliptin from Human Plasma-Applied to Biological Samples from Healthy Human Volunteers
}

\author{
Shereen Mowaka ${ }^{1,2,3}$, Nermeen Ashoush ${ }^{2,4}$, Mariam Tadros ${ }^{5}$, Noha El Zahar ${ }^{5}$ (i) \\ and Bassam Ayoub 1,2,*(D) \\ 1 Pharmaceutical Chemistry Department, Faculty of Pharmacy, The British University in Egypt, \\ El-Sherouk City, Cairo 11837, Egypt; shereen.hassib@bue.edu.eg \\ 2 The Center for Drug Research and Development (CDRD), Faculty of Pharmacy, The British University in \\ Egypt, El-Sherouk City, Cairo 11837, Egypt; nermeen.ashoush@bue.edu.eg \\ 3 Analytical Chemistry Department, Faculty of Pharmacy, Helwan University, Ain Helwan, Cairo 11795, Egypt \\ 4 Clinical Pharmacy and Pharmacy Practice Department, Faculty of Pharmacy, The British University in Egypt, \\ El-Sherouk City, Cairo 11837, Egypt \\ 5 Pharmaceutical Analytical Chemistry Department, Faculty of Pharmacy, Ain Shams University, \\ Organization of African Unity Street, Abassia, Cairo 11566, Egypt; \\ mariam.tadros@pharma.asu.edu.eg (M.T.); nohamorsyelzahar@pharma.asu.edu.eg (N.E.Z.) \\ * Correspondence: bassam.ayoub@bue.edu.eg; Tel.: +20-226890000 or +20-1225104337; Fax:+20-226300010
}

Academic Editor: Roberto Mandrioli

Received: 13 August 2020; Accepted: 12 September 2020; Published: 15 September 2020

\begin{abstract}
Enhancing drug extraction from human plasma is a challenging approach that critically affects pharmacokinetic and any further clinical studies based on the drug $C_{\min }$ and $C_{\max }$ values. It also has a serious impact on the sensitivity and the lower limit of quantification (LLOQ) value of the bio-analytical methods. An advanced liquid chromatography tandem mass spectrometry (LC-MS/MS) bio-analytical method of omarigliptin (25-1000 nM) was established in human plasma using one-step liquid-liquid extraction. Alogliptin was used as an internal standard (IS) to attain good recovery and reproducibility while reducing the effects of the matrix. Enhanced plasma extraction of omarigliptin was successfully achieved with tertiary butyl methyl ether-diethyl ether (TBME-DEE) mixture as the extracting solvent, while using acetonitrile as the diluent solvent for the IS to effectively decrease the formed emulsion. Multiple Reaction Monitoring (MRM) of the transition pairs of $m / z 399.2$ to 153.0 for omarigliptin and $m / z 340.2$ to 116.0 for alogliptin was employed in positive Electro Spray Ionization (ESI) mode. Human plasma samples were collected after $1.5 \mathrm{~h}\left(\mathrm{t}_{\max }\right)$ of Marizev ${ }^{\circledR}(12.5 \mathrm{mg})$ tablets administration to healthy human volunteers showing average concentration of $292.18 \mathrm{nM}$. Validation results were all satisfactory including successful stability studies with bias below $12 \%$. The proposed study will be valuable for ethnicity comparison studies that will be commenced on omarigliptin in Egypt by the authors in prospective study, following the FDA recommends, to evaluate possible sub-group dissimilarities that include pharmacokinetic parameters.
\end{abstract}

Keywords: enhanced extraction; human plasma; human volunteers; LC-MS/MS; omarigliptin

\section{Introduction}

Diabetes Mellitus prevalence increases constantly worldwide. Moreover, it is worthy to mention that many recent COVID-19 studies reported that Diabetes Mellitus was a major contributing factor either for non-survivals and/or hospitalization. Diabetic patients represented $12 \%$ of non-survivors in a study and represented $22 \%$ of the hospitalized patients in another study [1,2]. Generally, people with Diabetes Mellitus are most likely to suffer from different complications when infected with virus 
ranging from mild to severe. Unmanaged levels of blood glucose elevate the risk and the severity of any common respiratory attack [3,4]. Uncontrolled Diabetes Mellitus leads to a weak immunity response, as the body becomes no more able to fight the infection [1,2]. Hence, it becomes imperative to find better therapeutic strategies for glycemic control.

Gliptins are considered to be effective agents for the treatment of type 2 Diabetes Mellitus. Omariglitpin (OTN), Figure 1, is a long-acting once weekly administered antidiabetic drug acting as dipeptidyl peptidase-4 (DPP-4) inhibitor [5-7]. It has been licensed for use in Japan since 2015 but its phase III development in US has been halted for undisclosed commercial reasons [5]. It improved the glycated hemoglobin $\mathrm{A} 1 \mathrm{c}(\mathrm{HbA} 1 \mathrm{c})$ as it reversibly inhibits DPP-4 enzyme, which prolongs the circulating half-life of glucagon-like peptide-1 (GLP-1) increasing insulin secretion. Contrary to the once-daily administered DPP-4 inhibitors available in market, once-weekly administered OTN can improve patients' compliance to the treatment protocol [5-9].

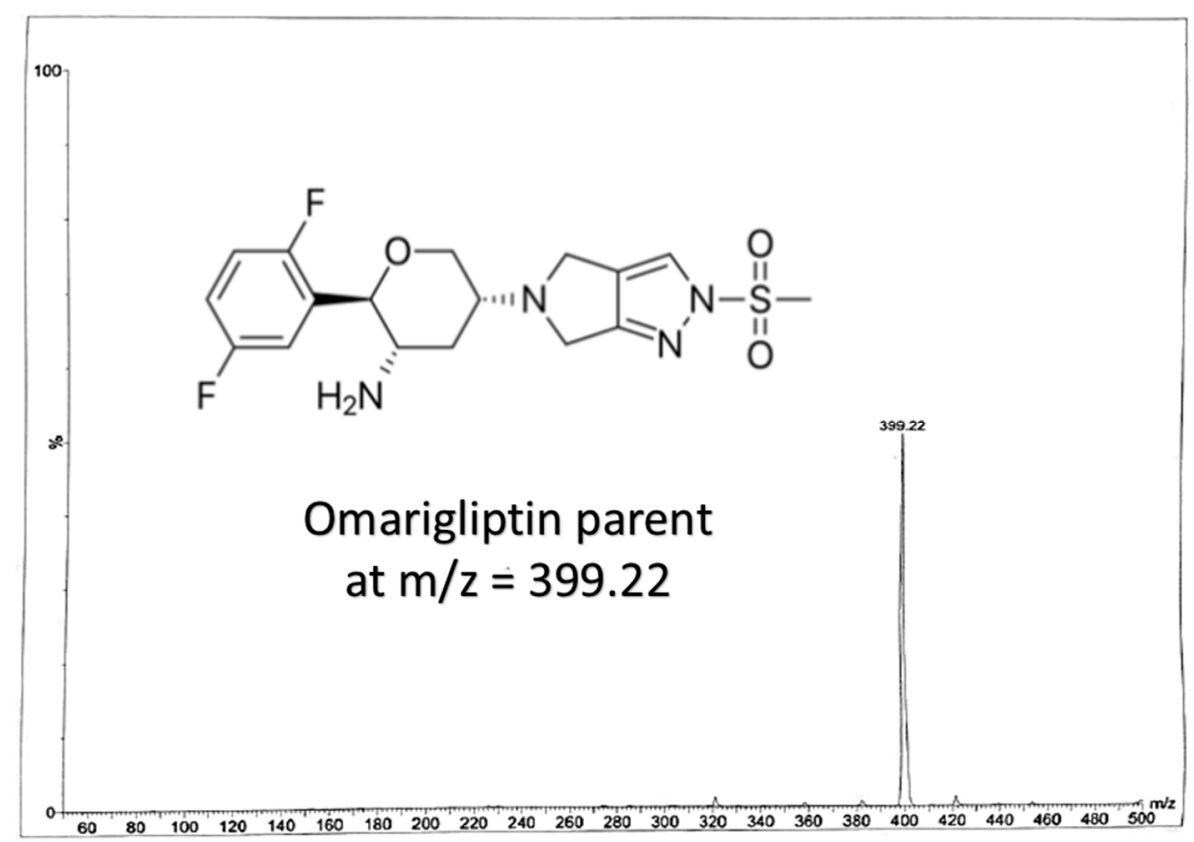

Figure 1. Chemical structure of omarigliptin and its full scan mass spectrum.

Literature review reveals that OTN pharmacokinetics parameters were studied with renal impairment [10], in repositioning studies [11] and in healthy Japanese volunteers [12]. OTN pharmacokinetics were investigating the supra-therapeutic dose of OTN [13] and the effect of age, sex and obesity [14]. Clinical studies were also concerned with the effects of multiple and chronic dosage regimens $[15,16]$. Although, these many articles deal with the pharmacokinetics and/or bio-analysis of OTN [10-16], most of the reported studies did not discuss the full details of the validated bio-analytical procedure that may be attributed to using the same methods for many clinical studies by the same common authors [12-17]. They were emphasizing the findings of the main pharmacokinetic parameters rather than developing or validating a bio-analytical procedure. One liquid chromatography tandem mass spectrometry (LC-MS/MS) method was discussed in full details for OTN bio-analysis [18] but that method was related to rats' plasma only and the authors used only direct precipitation with acetonitrile for the extraction of OTN from spiked and biological rats' plasma samples. The authors of the current study modified the previously mentioned rats' plasma method [18] in another repositioning publication [11] but with direct precipitation with acetonitrile. The lower limit of quantification (LLOQ) was $50 \mathrm{ng} / \mathrm{mL}$ which is very high in comparison to the LLOQ in the current work with the enhanced extraction technique. Moreover, the same authors reported in another UPLC study that the UV detector failed to detect OTN in human plasma due to interference [19]. 
Enhancing the extraction techniques for new drugs enrich the literature and opens the door for further studies. Hence, the purpose of our study is to focus on the bio-analysis of OTN using LC-MS/MS by means of an enhanced one-step liquid-liquid extraction of OTN from human plasma followed by vacuum evaporation and then reconstitution. The bioanalytical method was validated as per FDA guidelines to include linearity, selectivity, accuracy, precision, stability and matrix factor [20].

\section{Methods}

\subsection{Chemicals and Reagents}

Six different batches of blank human plasma, OTN raw material (99.0\%), Alogliptin raw material, internal standard (IS) $(99.2 \%)$, Marizev ${ }^{\circledR}$ (12.5 mg) tablets, tertiary butyl methyl ether (TBME), diethyl ether (DEE) were kindly donated by the British University in Egypt (CDRD, BUE) based on previous research collaborations (Repositioning CDRD-BUE project). Acetonitrile, methanol, and water (all of HPLC grade) and formic acid were obtained from (Sigma, St. Louis, MO, USA).

\subsection{LC-MS/MS Conditions}

Some of the coming conditions were adopted from the authors' previous LC-MS/MS work (published repositioning study on OTN and IS using direct precipitation for rats' plasma [11]). LC-MS/MS was performed via Waters ${ }^{\circledR}$ UPLC-TQ with Electro Spray Ionization (ESI) (USA), Mass Lynx software (4.1 version) and Agilent $\mathrm{C}_{18}$ column $(1.8 \mu \mathrm{m}, 50 \times 2.1 \mathrm{~mm})$. An isocratic mobile phase of acetonitrile/0.3\% formic acid $(90: 10, v / v)$, filtered via a $0.2 \mu \mathrm{m}$ filter membrane degassed for $25 \mathrm{~min}$, ten microliters as the injection volume, $0.3 \mathrm{~mL} / \mathrm{min}$ as the selected flow rate to decrease the retention time and provide a faster method and $1.2 \mathrm{~min}$ as the run time for OTN bioassay, were applied. The column temperature was set at $25^{\circ} \mathrm{C}$. The mass spectrometer parameters included the adjustment of cone voltage values to $40 \mathrm{~V}$ and $30 \mathrm{~V}$ and collision energy values to $50 \mathrm{eV}$ and $55 \mathrm{eV}$ for OTN and IS, respectively. Multiple Reaction Monitoring (MRM) of $m / z 399.2$ to 153.0 for OTN and $m / z 340.2$ to 116.0 for IS in the ESI positive mode was implemented. Further MS parameters were adjusted including turbo ions spray at $400{ }^{\circ} \mathrm{C}$, capillary temperature at $275^{\circ} \mathrm{C}$, sheath and auxiliary gas at 15 and 2 psi, respectively, ion spray voltage of $3800 \mathrm{~V}$, capillary voltage of $4 \mathrm{KV}$, capillary offset of 35 and desolvating line temperature at $400{ }^{\circ} \mathrm{C}$.

\subsection{Calibrators, QC Samples and Sample Preparation}

OTN standard stock solution in methanol was prepared as $(1 \mathrm{mM})$ then working solutions with different concentrations were prepared in methanol $(2.5,5,10,40,50,70,80$ and $100 \mu \mathrm{M}) .10 \mu \mathrm{L}$ of each one of the prepared working solutions was used to spike $990 \mu \mathrm{L}$ blank plasma to prepare calibrators and QC samples as $25 \mathrm{nM}$ (LLOQ), 50, 100, 400, $500 \mathrm{nM}$ (Medium quality control sample, MQC) and 700,800 (High quality control sample, HQC) and $1000 \mathrm{nM}$. An aliquot of $250 \mu \mathrm{L}$ of each plasma sample was spiked with $100 \mu \mathrm{L}$ of the IS in acetonitrile $(300 \mathrm{nM})$, liquid-liquid extracted by $1.5 \mathrm{~mL}$ of a mixture $(50: 50, v / v)$ of TBME and DEE for $15 \mathrm{~min}$ centrifugation at $15,000 \mathrm{rpm}$ and then frozen at $-80^{\circ} \mathrm{C} .1 .3 \mathrm{~mL}$ of the upper organic level was vacuum evaporated till dryness, reconstituted with $250 \mu \mathrm{L}$ methanol and injected into the auto-sampler. Peak area ratios to IS was used against concentrations to predict the calibration curve and the regression equation.

\subsection{Bioanalytical Validation}

FDA guidelines [20] were followed for 6 calibrators to predict the linearity while LLOQ, MQC, HQC levels $(n=5)$ were used to evaluate both accuracy and precision five times a day and on three successive days. The bias value, standard deviation (S.D.) and \% RSD were calculated. Six different batches of blank plasma (from different sources) donated form (CDRD-BUE) were checked for interference as a measure of selectivity. Injection of high values of concentration directly after the blank samples was used to ensure the absence of a significant carry over. Matrix factor was estimated 
by calculating the ratio of the peak area in the presence of the matrix components of human plasma (blank matrix as human plasma sample spiked after extraction with the drug under investigation, OTN), to the peak area in absence of the human plasma matrix components (solution of the drug, OTN). Comparing the area ratios under the peak of the post extracted samples to the neat standards was used to calculate the matrix factor while comparing the area ratios under the peak of the underlying extracted samples to the post extracted samples was used to calculate the extraction recovery. Stability of LLOQ and HQC was estimated based on 4 different bio-assays after leaving the samples $3 \mathrm{~h}$ in the auto-sampler or leaving them $3 \mathrm{~h}$ at room temperature (bench top stability) or analyzing them after three complete cycles of both freeze and thaw. Finally, stability for the long term was investigated by checking the samples after two weeks while freezing at $-80^{\circ} \mathrm{C}$.

\subsection{Biological Samples and Ethical Approval}

After the approval of the British University in Egypt ethical committee (Code: CL/2003, March 2020) and after submission of the proposed LC-MS/MS study to clinicaltrials.gov (ID: NCT04365907), one mL blood samples from 4 volunteers were collected after $1.5 \mathrm{~h}$ of the oral administration of the drug (Marizev $^{\circledR}$ as $12.5 \mathrm{mg}$ of OTN). Then, centrifugation at $3000 \mathrm{rpm}$ was performed to extract the plasma. This experimental trial was performed in order to employ the developed method for the determination of OTN in human plasma.

\section{Results and Discussion}

The proposed study will be valuable for ethnicity comparison studies that will be commenced on OTN in Egypt by the authors in a prospective study. This study will cover one week bio-analytical assays after its administration as the FDA recommends [20] and will compare results across pharmacokinetic studies to evaluate possible sub-group dissimilarities. Ethnic difference has been taken in consideration in recent years. Typically, global companies start clinical development in Japan after the United States and Europe, some genetic factors could explain a significant proportion of dose variability of many drugs between different ethnic groups [21,22]. The recognition of racial differences in disease outcomes and many investigations have identified genetic factors that explain a significant proportion of dose variability. This evidence underpins the prevailing hypothesis that genotype guided therapy should improve dosing accuracy [21,22] that requires validated bioanalytical studies to be used for the comparative pharmacokinetic studies. Therefore, an advanced analytical technique and enhanced extraction of drugs from human plasma become a challenging approach that greatly affects pharmacokinetics, other clinical studies based on the drug $C_{\min }$ and $C_{\max }$ values and the bio-analytical methods sensitivity.

In our study, effective liquid-liquid extraction based on the use of TBME-DEE mixture as the extracting solvent and vacuum evaporation followed by reconstitution, was established for the extraction of OTN from human plasma. Enhanced extraction was successfully achieved after using acetonitrile as the diluent solvent for the IS decreasing the emulsion formed due to the addition of the aqueous immiscible organic solvent TBME [23]. Therefore, using an appropriate mixture of the extracting solvent accompanied with high volume of acetonitrile; decreased the formed emulsion especially after freezing, which enabled the accurate withdrawing of up to $1.3 \mathrm{~mL}$ from the upper clear organic layer without interference from the emulsion intermediate layer. Adding acetonitrile (that contained the IS) was crucial as it decreased the usual resulting emulsion from mixing the immiscible extracting organic solvent and the plasma sample. Changing the ratio of the TBME-DEE mixture to $(60-40 \%)$ or $(40-60 \%)$, respectively did not affect the readings in the preliminary trials. LC-MS/MS bio-assay of OTN (25-1000 nM) was achieved in human plasma using alogliptin as IS. MRM function of the transition pairs of $m / z 399.2$ to 153.0 for OTN and $m / z 340.2$ to 116.0 for alogliptin was employed utilizing positive ESI mode, as shown in Figures 1-4. The transition pairs were also used to verify the identity of the analyte whereas no other qualifying ion transitions were detected or found significant to be used. The use of glass tubes was avoided throughout the investigation especially for 
vacuum evaporation, centrifugation and reconstitution to avoid the reported gliptins' adhesion [24] based on previous work by the authors on different gliptins in lab. The authors mentioned in the current study modified the rats' plasma method [18] in another repositioning publication [11] but with direct precipitation with acetonitrile, the LLOQ was $50 \mathrm{ng} / \mathrm{mL}$ which is very high in comparison to the LLOQ in the current work with the enhanced extraction technique $(9.98 \mathrm{ng} / \mathrm{mL}$ equivalent to $25 \mathrm{nM})$. The LLOQ in the current investigation is less than five times of the previous LLOQ [11]. Moreover, the same authors in another UPLC study [19], showed that the UV detector failed to detect OTN in human plasma due to interference from plasma endogenous components with the UV detector. They recommended LC-MS/MS accompanied with liquid-liquid extraction/vacuum evaporation to enhance the extraction procedure which is the case in the current study. A comparison between all the previously reported methods for OTN extraction and determination in plasma either rats' or human plasma, as shown in Table 1, was implemented. In spite of some common authors reported lower LLOQ $(2 \mathrm{ng} / \mathrm{mL}) \mathrm{in}$ human plasma using ethyl acetate (after pH adjustment) in liquid-liquid extraction [12-17], they did not mention the full validation study for the bioanalytical procedure. No data regarding stability studies, selectivity, carry over, extraction recovery, method development, full detailed chromatographic procedure and/or matrix effect was reported. Furthermore, the authors of the current investigation did not prefer to use the $\mathrm{pH}$ adjustment step that was reported in the previous LC-MS/MS method [12-17] as it did not show significant difference after using TBME-DEE mixture with the satisfying full validation bioanalytical results.

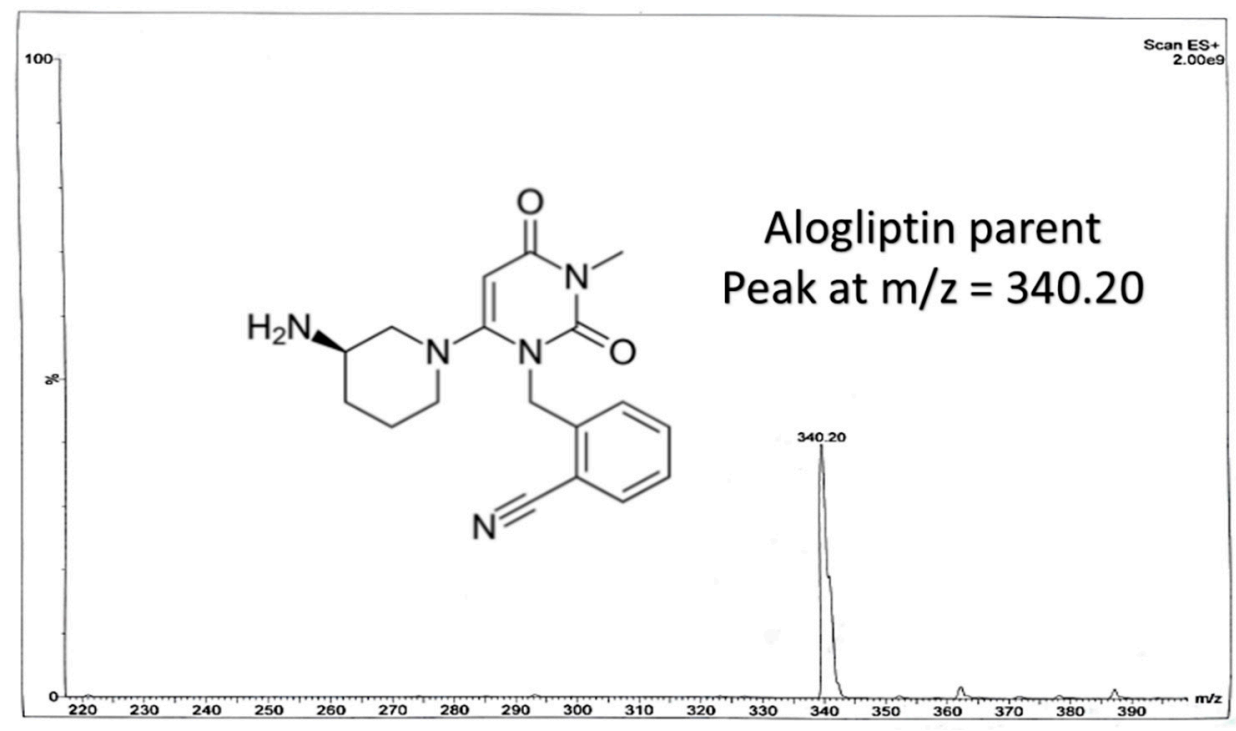

Figure 2. Chemical structure of alogliptin (IS) and its full scan mass spectrum. 


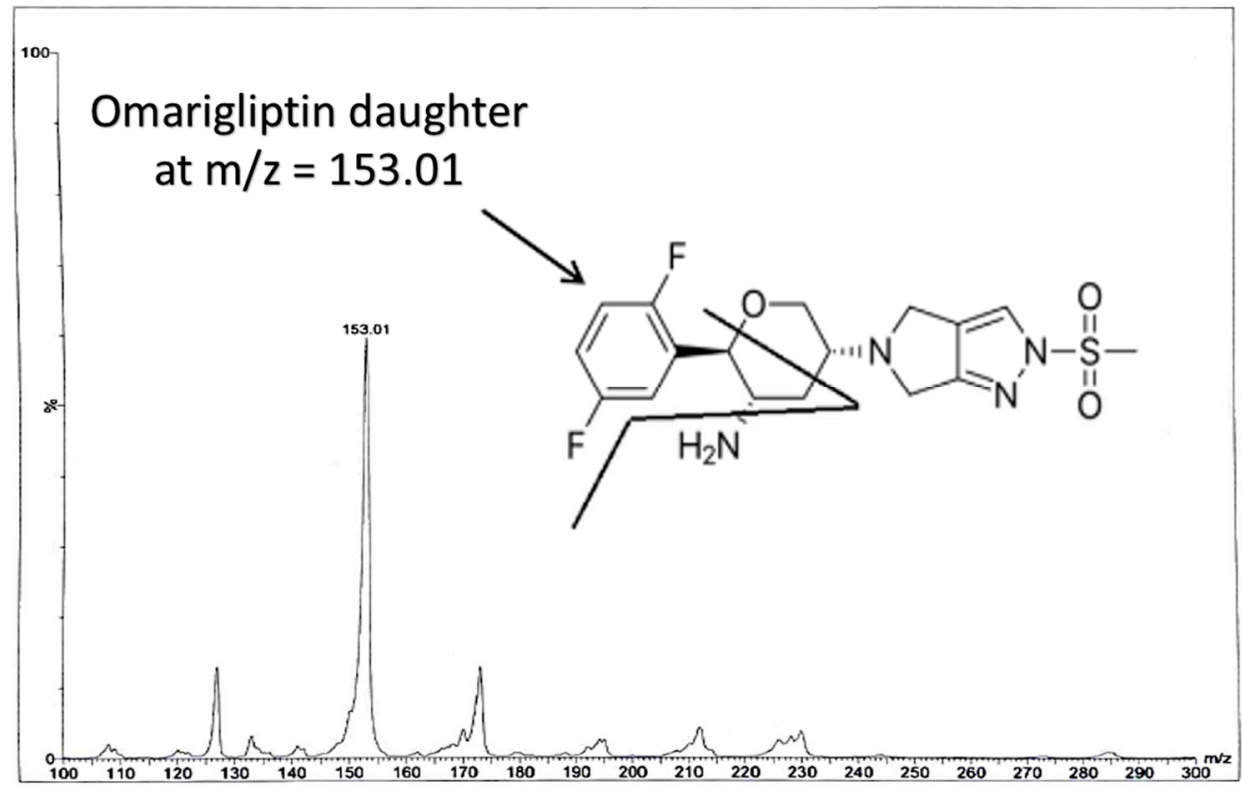

Figure 3. Daughter ions mass spectra in positive Electro Spray Ionization (ESI) ion detection mode with the proposed fragments showing $\mathrm{m} / \mathrm{z}$ at 153.01 for omarigliptin.

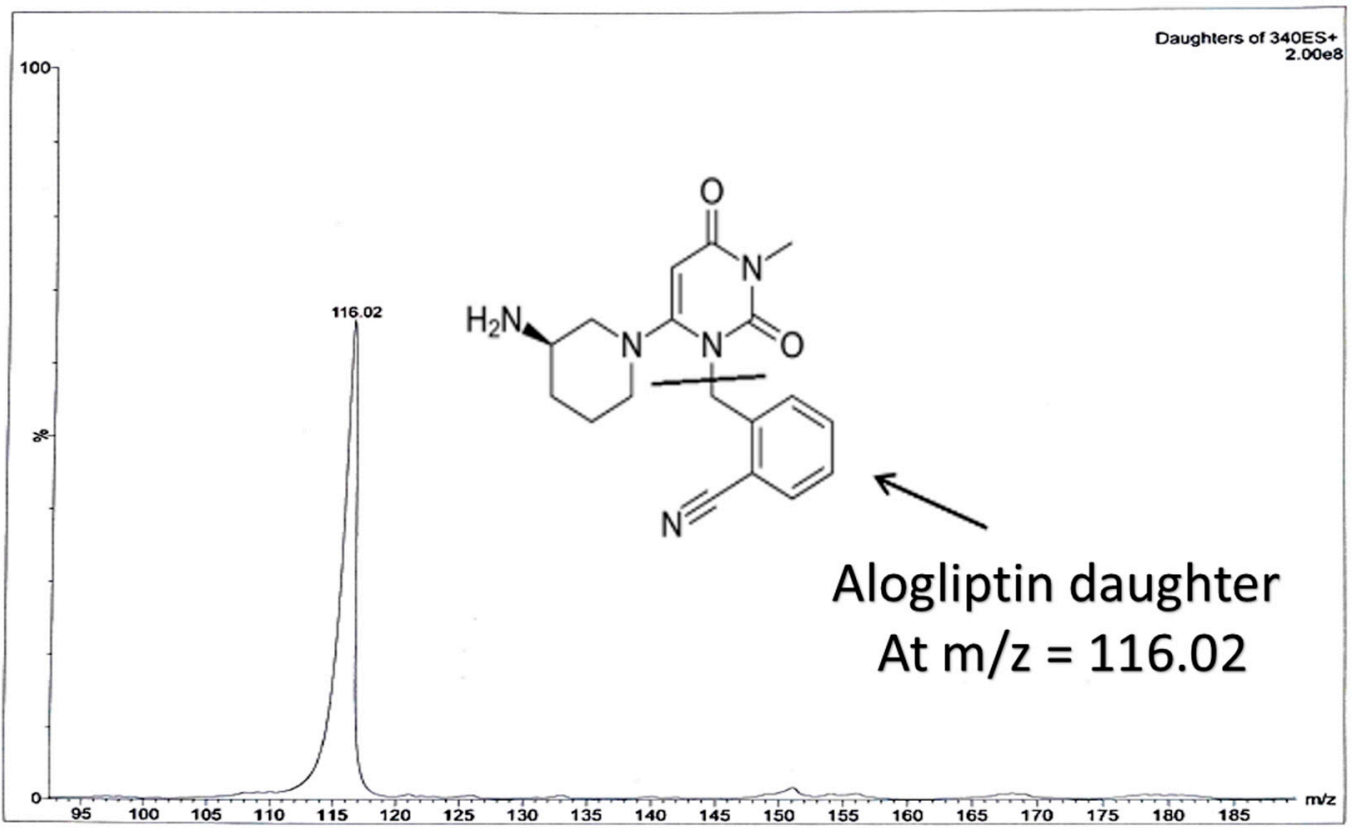

Figure 4. Daughter ions mass spectra in positive ESI ion detection mode with the proposed fragments showing $m / z$ at 116.02 for alogliptin.

Table 1. Comparison between previously reported methods for omariglitpin (OTN) extraction and determination in plasma either rats' plasma or human plasma.

\begin{tabular}{lcccc}
\hline Method & Extraction & LLOQ & Application & Reference \\
\hline LC-MS/MS & Liquid-Liquid using TBME-DEE & $25 \mathrm{nM}(9.96 \mathrm{ng} / \mathrm{mL})$ & Human plasma & Underlying investigation \\
\hline LC-MS/MS & Direct precipitation using Acetonitrile & $50 \mathrm{ng} / \mathrm{mL}$ & Rats' plasma & [11] \\
\hline LC-MS/MS & Liquid-Liquid using Ethyl acetate after $\mathrm{pH}$ adjustment & $2 \mathrm{ng} / \mathrm{mL}$ & Human plasma & {$[12-17]$} \\
\hline LC-MS/MS & Direct precipitation using Acetonitrile & $4 \mathrm{ng} / \mathrm{mL}$ & Rats' plasma & [18] \\
\hline UPLC-UV & Liquid-Liquid using DEE & $2.5 \mu \mathrm{m} / \mathrm{mL}$ & Rats' plasma & [19] \\
\hline
\end{tabular}


Calibration (25-1000 nM) and full detailed validation outcomes were satisfactory with FDA bio-analytical guidelines [20]. Adequate method selectivity from six different batches of blank plasma was designated where no significant interference was observed among the MRM channels in blank (Figure 5), zero samples with IS (Figure 6) and reasonable outcomes at the LLOQ level of $25 \mathrm{nM}$ (Figure 7) and no significant carry over was detected. The authors used LC-MS/MS technique as a detection technique rather than the separation strategy and based on their experience with chromatography, they ensured, while developing the LC method, that both the drug and the IS did not elute on the dead volume time before applying the sequence order in the instrument. The equation of the calibration curve was; $y=0.0033 x-0.0701, r=0.9998$ showing the good linearity of the applied method. Accuracy $(n=5)$ and precision $(n=15)$ were within $\pm 20 \%$ as shown in (Table 2). Extraction recovery was $87.5 \%$ for the LLOQ and $89.66 \%$ for the HQC sample. Matrix factor denoting the effect of the matrix on the signal response and the ionization efficiency through matrix enhancement and/or suppression was evaluated. It was ranged from $81.4 \%$ to $86.24 \%$ indicating ion suppression for all concentrations of OTN in plasma. Stability measurements mentioned under methods showed recoveries more than $85 \%$ from the time-zero control which indicates OTN stability can be maintained through the sample treatment and storage.
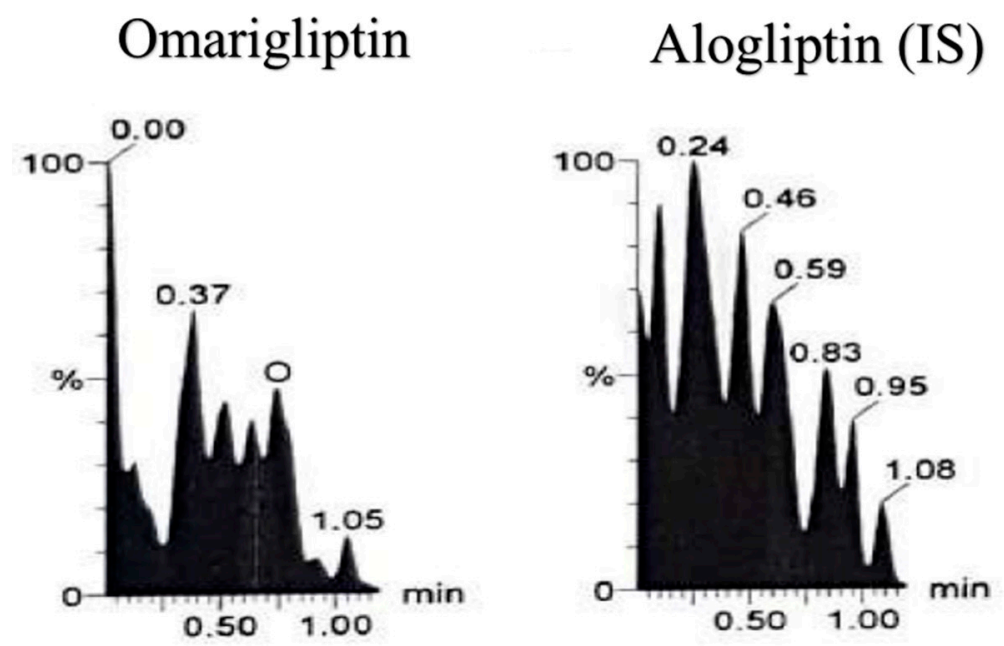

Figure 5. Blank plasma sample using liquid chromatography tandem mass spectrometry (LC-MS/MS).

\section{Omarigliptin}
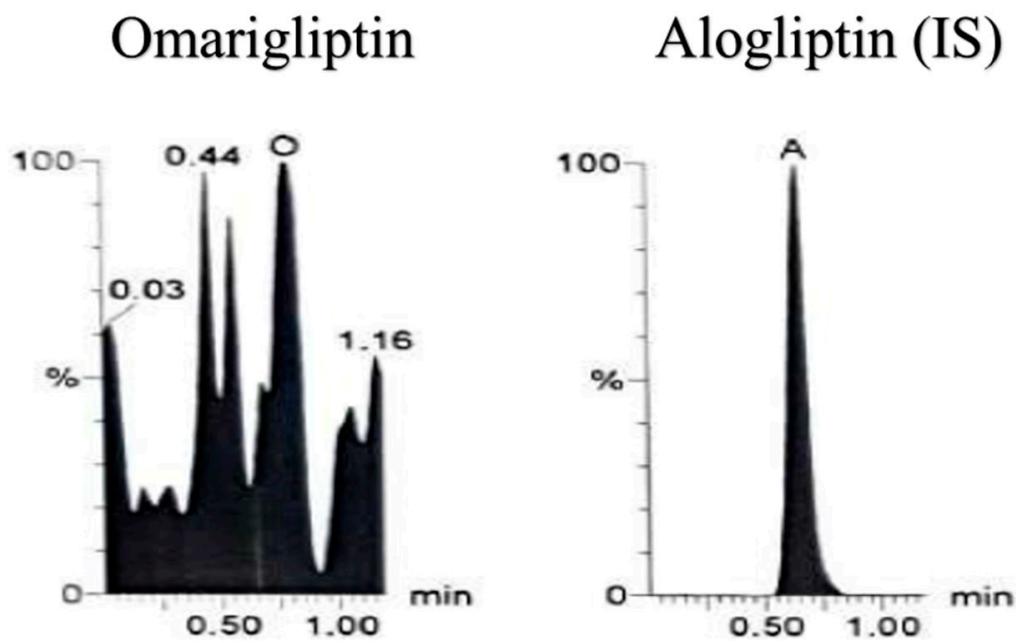

Figure 6. Zero plasma sample (spiked with IS) using LC-MS/MS showing only the IS using Multiple reaction monitoring (MRM) chromatogram of omarigliptin $(m / z=399.2$ to 153.0) and alogliptin (IS, $m / z=340.2$ to 116.0 ). 
Omarigliptin

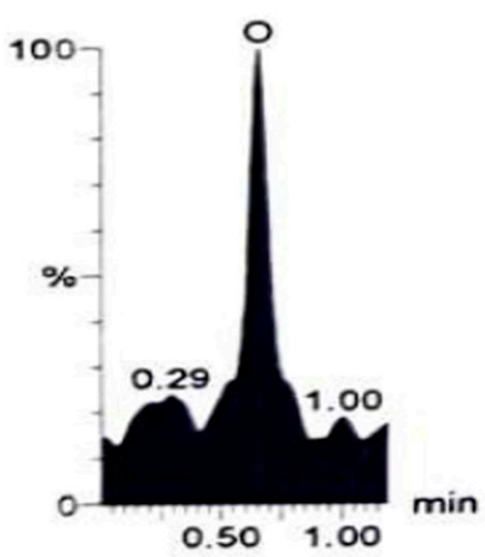

\section{Alogliptin (IS)}

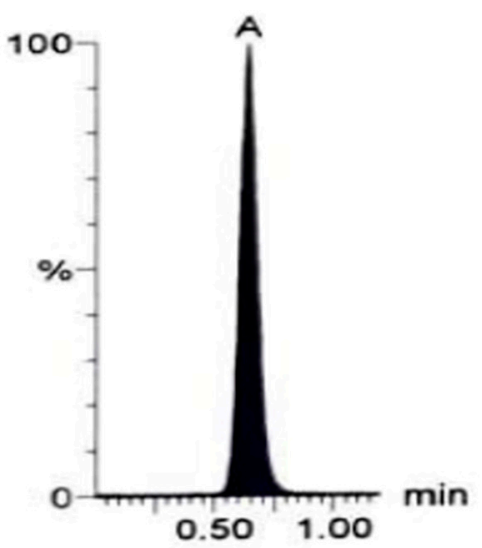

Figure 7. Lower limit of quantification (LLOQ) plasma sample using LC-MS/MS (25 nM of Omarigliptin) using Multiple reaction monitoring (MRM) chromatogram of omarigliptin $(m / z=399.2$ to 153.0$)$ and alogliptin (IS, $m / z=340.2$ to 116.0 ).

Table 2. Accuracy and precision results for OTN determination by the proposed LC-MS/MS method.

\begin{tabular}{|c|c|c|c|c|}
\hline \multicolumn{2}{|c|}{ Accuracy and Precision ( $n=5$, Three days) } & LLOQ (25 nM) & MQC (500 nM) & HQC (800 nM) \\
\hline \multirow{4}{*}{ 1st day Intraday } & Bias (mean, $n=5)$ & 15.41 & -6.30 & -1.09 \\
\hline & Average Percent Recovery & $115.41 \%$ & $93.70 \%$ & $98.91 \%$ \\
\hline & S.D. & 14.55 & 2.01 & 3.57 \\
\hline & \% R.S.D & 12.61 & 2.14 & 3.64 \\
\hline \multirow{4}{*}{ 2nd day Intraday } & Bias (mean, $n=5$ ) & 10.34 & 1.49 & -0.10 \\
\hline & Average Percent Recovery & $110.34 \%$ & $101.49 \%$ & $99.90 \%$ \\
\hline & S.D. & 15.37 & 1.90 & 1.82 \\
\hline & \% R.S.D & 13.92 & 1.87 & 1.82 \\
\hline \multirow{4}{*}{ 3rd day Intraday } & Bias $($ mean, $n=5)$ & 13.80 & 0.98 & -0.91 \\
\hline & Average Percent Recovery & $113.80 \%$ & $100.98 \%$ & $99.09 \%$ \\
\hline & S.D. & 9.57 & 1.83 & 2.62 \\
\hline & \% R.S.D & 8.41 & 1.81 & 2.64 \\
\hline \multirow{4}{*}{ Interday } & Bias (mean, $\mathrm{n}=15)$ & 13.40 & -1.30 & -1.01 \\
\hline & Average Percent Recovery & $113.40 \%$ & $98.70 \%$ & $98.99 \%$ \\
\hline & S.D. & 13.41 & 4.02 & 2.89 \\
\hline & \% R.S.D & 11.83 & 4.07 & 2.92 \\
\hline
\end{tabular}

Eventually, OTN validated bioanalytical method was efficaciously applied to analyze human plasma samples. They were collected after $1.5 \mathrm{~h}\left(\mathrm{t}_{\max }\right)$ of Marizev ${ }^{\circledR}(12.5 \mathrm{mg})$ tablets administration to healthy human volunteers (as shown in Figure 8) showing average concentration of $292.18 \mathrm{nM}$, standard deviation of $21.1 \mathrm{nM}$ and Percent relative standard deviation of $7.22 \%$. 


\section{Omarigliptin}

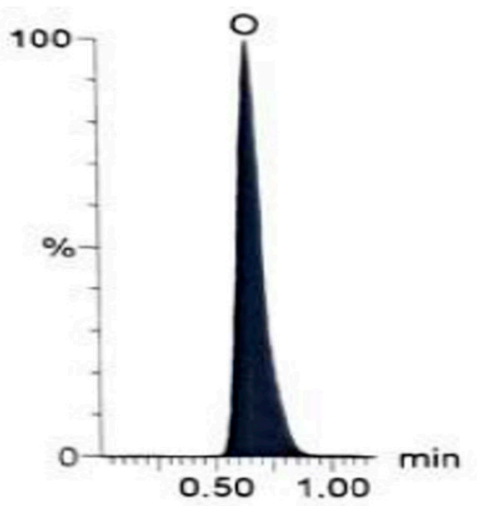

Alogliptin (IS)

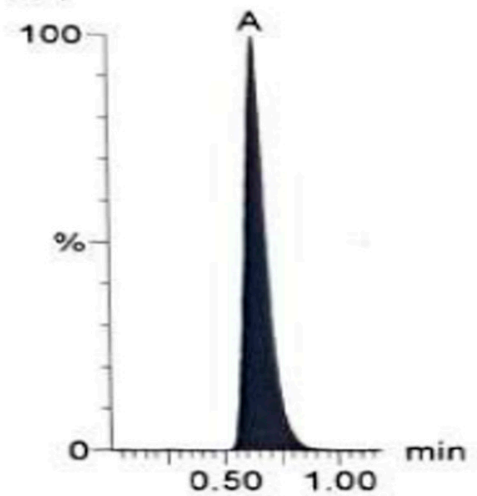

Figure 8. Multiple reaction monitoring (MRM) chromatogram of omarigliptin $(\mathrm{m} / \mathrm{z}=399.2$ to 153.0$)$ and alogliptin (IS, $m / z=340.2$ to 116.0 ) in human plasma sample obtained $1.5 \mathrm{~h}$ after oral administration of one Marizev ${ }^{\circledR}$ tablet $(12.5 \mathrm{mg})$.

\section{Conclusions}

Working on anti-diabetic drugs' bio-analysis is of great importance in the current days. It opens the door for ethnicity comparisons related to more pharmacokinetic bioequivalence studies and drugs' approval in more countries leading to better $\mathrm{HbA} 1 \mathrm{c}$ control and other outcomes. Bio-analysis of OTN (25-1000 nM) using LC-MS/MS was established as per FDA guidelines. Liquid-liquid extraction based on TBME-DEE mixture without the need of $\mathrm{pH}$ adjustment and vacuum evaporation followed by reconstitution, was implemented. Then, enhanced extraction of OTN from human plasma was achieved successfully after using acetonitrile as the diluent solvent for the IS to decrease the formed emulsion. The effective liquid-liquid extraction showed more sensitive results than direct precipitation. The LLOQ in the current investigation is less than five times of the previous LLOQ reported by the same authors. Enhancing the extraction techniques for new drugs enrich the literature and opens the door for further pharmacokinetic and bioequivalence studies.

Author Contributions: S.M., N.A., M.T., N.E.Z. and B.A. had equally contributed to the present bioanalytical work. All authors have read and agreed to the published version of the manuscript.

Funding: This research was partially funded by the British University in Egypt "Grant number YIRG-2019" granted to Dr. Shereen Hassib Mowaka from BUE as the PI with valuable contribution of all the other authors as researchers. Moreover, the APC was kindly funded by the British University in Egypt.

Conflicts of Interest: The authors declare no conflict of interest.

\section{References}

1. Yang, X.; Yu, Y.; Xu, J.; Shu, H.; Xia, J.; Liu, H.; Wu, Y.; Zhang, L.; Yu, Z.; Fang, M.; et al. Clinical course and outcomes of critically ill patients with SARS-CoV-2 pneumonia in Wuhan, China: A single-centered, retrospective, observational study. Lancet Respir. Med. 2020, 2600, 30079-30085. [CrossRef]

2. Gupta, R.; Ghosh, A.; Singh, A.K.; Misra, A. Clinical considerations for patients with diabetes in times of COVID-19 epidemic. Diabetes Metab. Syndr. Clin. Res. Rev. 2020, 14, 211-212. [CrossRef] [PubMed]

3. Yang, J.K.; Feng, Y.; Yuan, M.Y.; Yuan, S.Y.; Fu, H.J.; Wu, B.Y.; Sun, G.Z.; Yang, G.R.; Zhang, X.L.; Wang, L.; et al. Plasma glucose levels and diabetes are independent predictors for mortality and morbidity in patients with SARS. Diabet. Med. 2006, 23, 623-628. [CrossRef] [PubMed]

4. Schoen, K.; Horvat, N.; Guerreiro, N.F.C.; de Castro, I.; de Giassi, K.S. Spectrum of clinical and radiographic findings in patients with diagnosis of $\mathrm{H} 1 \mathrm{~N} 1$ and correlation with clinical severity. BMC Infect. Dis. 2019, 19, 964. [CrossRef]

5. Galloway, I.; McKay, G.; Fisher, M. Omarigliptin. Pract. Diabetes 2017, 34, 70-71. [CrossRef]

6. Tan, X. Omarigliptin for the treatment of type 2 diabetes. Endocrine 2016, 54, 24-31. [CrossRef] 
7. Wang, X.; Li, X.; Qie, S.; Zheng, Y.; Liu, Y.; Liu, G. The efficacy and safety of once-weekly DPP-4 inhibitor omarigliptin in patients with type 2 diabetes mellitus A systemic review and meta-analysis. Medicine 2018, 97, e11946. [CrossRef]

8. Burness, C.B. Omarigliptin: First Global Approval. Drugs 2015, 75, 1947-1952. [CrossRef]

9. Ito, Y.; Mori, M.; Matsumoto, Y.; Okamoto, T. Pharmacological action and clinical results of omarigliptin (MARIZEV ${ }^{\circledR}$ tablet), a novel dipeptidyl peptidase-4 inhibitor for once-weekly treatment of Type 2 diabetes. Folia Pharmacol. Jpn. 2017, 149, 128-137. [CrossRef]

10. Jain, L.; Chain, A.S.Y.; Tatosian, D.A.; Hing, J.; Passarell, J.A.; Kauh, E.A.; Lai, E. Pharmacokinetic-pharmacodynamic (dipeptidyl peptidase-4 inhibition) model to support dose rationale in diabetes patients, including those with renal impairment, for once-weekly administered omarigliptin. Br. J. Clin. Pharmacol. 2019, 85, 2759-2771. [CrossRef]

11. Ayoub, B.M.; Mowaka, S.; Safar, M.M.; Ashoush, N.; Arafa, M.G.; Michel, H.E.; Tadros, M.M.; Elmazar, M.M.; Mousa, S.A. Repositioning of omarigliptin as a once-weekly intranasal anti-parkinsonian agent. Sci. Rep. 2018, 8, 8959. [CrossRef]

12. Tsuchiya, S.; Friedman, E.; Addy, C.; Wakana, A.; Tatosian, D.; Matsumoto, Y.; Suzuki, H.; Kauh, E. Single and multiple dose pharmacokinetics and pharmacodynamics of omarigliptin, a novel, once-weekly dipeptidyl peptidase-4 inhibitor, in healthy Japanese men. J. Diabetes Investig. 2017, 8, 84-92. [CrossRef] [PubMed]

13. Tatosian, D.A.; Cardillo Marricco, N.; Glasgow, X.S.; DeGroot, B.; Dunnington, K.; George, L.; Gendrano, I.N.; Johnson-Levonas, A.O.; Swearingen, D.; Kauh, E.A. Thorough QTc study confirms early pharmacokinetics/QTc Modeling: A supratherapeutic dose of omarigliptin, a once-weekly DPP-4 Inhibitor, does not prolong the QTc interval. Clin. Pharmacol. Drug Dev. 2016, 5, 383-392. [CrossRef] [PubMed]

14. Addy, C.; Tatosian, D.A.; Glasgow, X.S.; Iii, I.N.G.; Sisk, C.M.; Kauh, E.A.; Stoch, S.A.; Wagner, J.A. Effects of age, sex, and obesity on the single-dose pharmacokinetics of omarigliptin in healthy subjects. Clin. Pharmacol. Drug Dev. 2016, 5, 374-382. [CrossRef] [PubMed]

15. Addy, C.; Tatosian, D.; Glasgow, X.S.; Gendrano, I.N., III; Kauh, E.; Martucci, A.; Johnson-Levonas, A.O.; Selverian, D.; Matthews, C.Z.; Gutierrez, M.; et al. Pharmacokinetic and pharmacodynamic effects of multiple-dose administration of omarigliptin, a once-weekly Dipeptidyl Peptidase-4 Inhibitor, in obese Participants with and without type 2 diabetes mellitus. Clin. Ther. 2016, 38, 516-530. [CrossRef] [PubMed]

16. Krishna, R.; Addy, C.; Tatosian, D.; Glasgow, X.S.; Gendrano, I.N.; Robberechts, M.; Haazen, W.; de Hoon, J.N.; Depré, M.; Martucci, A.; et al. Pharmacokinetics and pharmacodynamics of omarigliptin, a once-weekly dipeptidyl peptidase-4 (DPP-4) inhibitor, after single and multiple doses in Healthy Subjects. J. Clin. Pharmacol. 2016, 56, 1528-1537. [CrossRef]

17. Xu, S.; Tatosian, D.; Mcintosh, I.; Caceres, M.; Matthews, C.; Samuel, K.; Selverian, D.; Kumar, S.; Kauh, E. Absorption, metabolism and excretion of [14C] omarigliptin, a once-weekly DPP-4 inhibitor, in humans. Xenobiotica 2018, 48, 584-591. [CrossRef]

18. Li, M.-F.; Hu, X.-X.; Ma, A.-Q. Ultra-high pressure liquid chromatography-tandem mass spectrometry method for the determination of omarigliptin in rat plasma and its application to a pharmacokinetic study in rats. Biomed. Chromatogr. 2017, 31, e3975. [CrossRef]

19. Attallah, M.A.; Mowaka, S.; Elkady, E.F.; Fouad, M.; Ayoub, B. Analysis and bio-analysis of omarigliptin, trelagliptin and alogliptin: Applied to biological samples and degradation kinetic study. Microchem. J. 2019, 148, 253-261. [CrossRef]

20. FDA Bioanalytical Method Validation, Guidance for Industry, U.S.Department of Health and Human Services Food and Drug Administration Center for Drug Evaluation and Research (CDER) Center for Veterinary Medicine (CVM). 2018. Available online: https://www.fda.gov/regulatory-information/search-fda-guidancedocuments/bioanalytical-method-validation-guidance-industry (accessed on 15 July 2020).

21. Fukunaga, S.; Kusama, M.; Arnold, F.L.; Ono, S. Ethnic differences in pharmacokinetics in new drug applications and approved doses in Japan. J. Clin. Pharmacol. 2011, 51, 1237-1240. [CrossRef]

22. Limdi, N.A.; Brown, T.M.; Yan, Q.; Thigpen, J.L.; Shendre, A.; Liu, N.; Hill, C.E.; Arnett, D.K.; Beasley, T.M. Race influences warfarin dose changes associated with genetic factors. Blood 2015, 126, 539-545. [CrossRef] [PubMed] 
23. Xue, Y.-J.; Pursley, J.; Arnold, M.E. A simple 96-well liquid-liquid extraction with a mixture of acetonitrile and methyl t-butyl ether for the determination of a drug in human plasma by high-performance liquid chromatography with tandem mass spectrometry. J. Pharm. Biomed. Anal. 2004, 34, 369-378. [CrossRef]

24. Suresh, P.S.; Srinivas, N.R.; Mullangi, R. A concise review of the bioanalytical methods for the quantitation of sitagliptin, an important dipeptidyl peptidase-4 (DPP4) inhibitor, utilized for the characterization of the drug. Biomed. Chromatogr. 2016, 30, 749-771. [CrossRef]

Sample Availability: Samples of the compounds are not available from the authors.

(C) 2020 by the authors. Licensee MDPI, Basel, Switzerland. This article is an open access article distributed under the terms and conditions of the Creative Commons Attribution (CC BY) license (http://creativecommons.org/licenses/by/4.0/). 